\title{
Multidisciplinary nexus for global public health challenges
}

\author{
Md. Aminul Haque ${ }^{1}$, Budi Aji ${ }^{2}$, Siwi Pramatama Mars Wijayanti ${ }^{2}$ \\ ${ }^{1}$ Department of Population Science, Faculty of Social Science, University of Dhaka, Dhaka, \\ Bangladesh \\ ${ }^{2}$ School of Public Health, Faculty of Health Sciences, Jenderal Soedirman University, \\ Purwokerto, Central Java, Indonesia
}

Corresponding author: Md. Aminul Haque, Email: aminul.haque@du.ac.bd

Disease pandemic has shown that human health does not belong to health human resources only but beyond it. People should realize that health issues could not only be relied on the health sector but it also needs a cross-cutting stakeholders such as social scientists, economists, politicians, non-governmental organizations, mass media, private entities, mass participation etc. So, human health belongs to the participation of everyone because it comes from everyone. No one will deny it!

The interdisciplinary approach is not a new idea for health issue, it however needs to be refined, redefined, and reformulated for its praxis in the real world. Bridging from theory to practice and collaborating scientists, policy makers and implementers. Urgent need for building a partnership based on empirical evidence to applied program (1). This requires an insight of academic perspective and flexibility of implementation in the field. This comes from the scientific paper to program report (2). As such, we are focusing our first issue of the 'Insights in Public Health Journal (IPHJ)' on multidisciplinary collaboration for global public health challenges.

To support the idea of multidisciplinary nexus, for example in this issue contains a variety topics dealing with communicable and non-communicable disease, health system issues, reproductive health, education and so on. This represents an interdisciplinary approach, combines multiperspectives for gaining upcoming new insights in public health challenges. Multidisciplinary addresses various needs to tackle health problems by using many perspectives and elaborates collective teams from a different level of stakeholders (3).

With respect to increase public awareness about multidisciplinary nexus on public health, in the case of this journal-title, we think about the essential of scientific role in public health practice by providing empirical evidences and knowledge for supporting policies and program implementation. Then, also promoting the implementation of evidence-based practice into public 
health services (4). At the same time, the Journal would persuade scientists to work closely together with implementers as part of collaborative nexus. IPHJ is an attempt responding to concerns about this need, therefore this journal will not only focusing on public health issues but also accepting cross-disciplinary papers related to health for gaining diversity of science.

Finally, the journal would open up windows for discussion about public health issues in many aspects and different perspectives from both local and global levels, so could produce beneficial insights in the future. We welcome all the scientists and researchers to contribute by sending letters, reviews and papers in response to this call. Your contribution will initiate an effective and a fruitful debates among scholars which are likely to enrich this scientific forum.

\section{Ethical issues}

Not applicable.

\section{Competing interests}

None.

\section{References}

1. Houry D, Swahn MH, Hankin A. Using a multidisciplinary approach for a multi-faceted public health problem. Western Journal of Emergency Medicine. 2013.

2. Bejean M, Guilloux G, Picard R, Pingaud H. Interdisciplinary perspectives. In: Connected Healthcare for the Citizen. 2018.

3. Dion X. A multidisciplinary team approach to public health working. British journal of community nursing. 2004.

4. Sandström B, Borglin G, Nilsson R, Willman A. Promoting the Implementation of EvidenceBased Practice: A Literature Review Focusing on the Role of Nursing Leadership. Worldviews Evidence-Based Nurs. 2011; 